\title{
ESTIMATING WITH BIM: A SURVEY OF US CONSTRUCTION COMPANIES
}

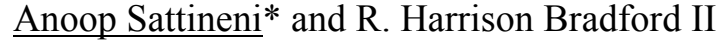 \\ McWhorter School of Building Science, Auburn University, Auburn, AL, USA \\ *Corresponding author (anoop@auburn.edu)
}

\begin{abstract}
The use of Building Information Modeling (BIM) in the construction industry is on the rise. It is widely acknowledged that adoption of BIM would cause a seismic shift in the business processes within the construction industry and related fields. Cost estimation is a key aspect in the workflow of a construction project. Processes within estimating, such as quantity survey and pricing, may be automated by using existing BIM software in combination with existing estimating software. The adoption of this combination of technologies is not as widely seen as might be expected. Researchers conducted a survey of construction practitioners to determine the extent to which estimating processes were automated in the conjunction industry, with the data from a BIM model. Survey participants were asked questions about how BIM was used within their organization and how it was used in the various tasks involved in construction cost estimating. The results of the survey data revealed that while most contractors were using BIM, only a small minority were using it to automate estimating processes. Most organizations reported that employees skilled in BIM did not have the estimating experience to produce working estimates from BIM models and vice-versa. The results of the survey are presented and analyzed to determine conditions that would improve the adoption of these new business processes in the construction estimating field.
\end{abstract}

Keywords: BIM, Estimating, Survey, US Construction Companies

\section{INTRODUCTION}

A new technology named Building Information Modeling (BIM) is fast establishing itself as the brand new approach to design-build-operate buildings in the twenty first century. The National Building Information Model Standard (NBIMS) defines [4] a building information model as “... a digital representation of physical and functional characteristics of a facility. As such it serves as a shared knowledge resource for information about a facility forming a reliable basis for decisions during its life-cycle from inception onward." Decades of efforts by academic, governmental and research institutions have resulted in this technology become a reality in the US ArchitecturalEngineering-Construction (AEC) sector.

It has been sufficiently established that advances in information technology provide unique competitive advantages to construction [5]. The construction industry benefits in several ways by adopting BIM technology as described by the NBIMS [4]. BIM has already been adopted by several contractors in the US and is quickly gaining popularity within the construction industry. The use of BIM technology is also being specified as a requirement by several facility owners including the U.S. Governmental Services Administration [11]. Advances within BIM technology are continuously increasing practical applications for the AEC industries [1].

BIM technology provides the means to deliver a better product for facility owners; however it also has wide ranging impacts for the construction industry. A construction company adopting BIM will have to carefully consider all the processes within the company. Processes such as cost estimating and scheduling have to be reengineered in light of the new technology. On the one hand new technology offers the lure of simplifying processes and providing immediate benefit but on the other, replacing existing technology has several costs associated 
with it. In the context of this paper, constructors must retrain their staff to perform estimating activities with the help of BIM technology. The task of training an entire estimating staff is expensive and the question of 'cost of change' is an important one that construction companies must carefully consider.

Cost estimation is essential to the processes in the construction industry. Cost estimating is the process of predicting the costs of a project based on the required materials, labor and time constraints; it is critical in construction projects for both budgeting and scheduling. According to a survey conducted by Sounder and Gier in 2006, estimating skills were rated as the number one skill industry professionals expect construction management graduates to possess. With the growing competitiveness of the construction industry, construction firms are seeking more efficient ways to estimate the cost of complex projects [6]. Contractors are attempting to speed the estimating process and limit administrative costs [3]. In a 2007 survey of eighty-six Finnish construction firms, seventy-six percent used BIM in some phase of design; yet, BIM was not being implemented for cost estimating [8].

Estimating currently lacks the guarantees of comprehension and consistency that BIM offers throughout the design and construction process [9]. With the traditional estimating methods, the architect will get a cost estimate at the mid- and end- points of each phase of the design process, since it takes up to three weeks for the cost consultant to generate each estimate [2]. These delays can be limited if BIM is implemented in cost estimating; Building Information Modeling generates rapid cost feedback throughout the building lifecycle when used for cost estimating [13]. BIM has the potential to allow construction companies to estimate the cost of a project with more detail and accuracy, while reducing time and expenses needed. BIM can make estimating simpler and more accurate, but requires the cooperation of the architect and engineer while designing the digital model [13]. Since Building Information Modeling recognizes actual materials used in construction, the BIM software has the potential to perform the quantity take-offs and pricing necessary for cost estimating. Often, the accuracy of cost estimating is positively correlated with the amount of information available on the project [10]. According to Tiwari et al. [13], "Model-based cost estimating is the process of integrating the object attributes from the 3D model of the designer with the cost information from database of the estimator." Using BIM to improve estimating methods will facilitate increased control of time and costs in a construction project.

Cost estimating is currently a time consuming process, requiring an entire team of estimators. There are multiple factors hindering the transition to model-based estimating; however, the risks are justified by the benefits of estimating with BIM. Building Information Modeling has the capability to automate a quantity take-off, which will reduce the time and costs required to estimate a project; however, the industry is not using BIM for estimating [13]. BIM software is compatible with estimating software, such as Innovaya Composer, which converts BIM files, making them compatible with Timberline's estimate and quantity data. Innovaya's Visual Estimating program links BIM data, consisting of doors, windows, and walls, etc., with Timberline's estimating data of labor costs, material costs, and equipment costs. By compiling the quantity data in a single database, Timberline can assign costs, automating the estimating process [7]. Although Building Information Modeling is widely discussed in the construction industry, there have been very few implementations using BIM for cost estimating. The most challenging aspect for the implementation of Building Information Modeling is the cultural switch within the company. Another aspect that is very important to implementing any new estimating software is the confidence of the estimator in the automation. Therefore any new software will have to undergo a thorough examination with several cross-checks. Estimators require one to two months of training with BIM software before the company will save time and costs on a project. Building Information Modeling allows estimators to decrease the time to complete their estimates, while increasing the estimate's accuracy [6]. The use of Building 
Information Modeling for estimating will not make estimators obsolete, as BIM will always require a human element to accurately price materials. Construction firms must look at BIM as an investment, where some time and money will have to be spent to save greater time and costs in the future [12].

\section{METHODOLOGY}

A web-based survey was conducted to analyze the construction industry's use of Building Information Modeling for cost estimating in the US. The survey was conducted to understand the role of BIM's to develop cost estimates for construction projects. Academic research included reviewing academic papers and testing BIM software and to examine the difficulties in switching from traditional estimating methods to estimating with Building Information Modeling.

Responses were categorized to identify whether participants were employed by small, medium, or large companies. The first step in the survey was to obtain information about the survey participants. Participants were asked the size of their company, their location, and their job description. The second category of questions related to BIM software, as participants were asked what BIM programs they are currently using, how estimating programs are being paired with BIM, and if BIM is being utilized for quantity take-off, pricing, and scheduling. Likert scale based questions were used to analyze the accuracy and difficulty in performing quantity take-offs with BIM. Survey participants were also asked if BIM improves the quality of estimates or decreases the amount of time required for cost estimating. The survey was completed by members of the construction industry throughout the United States. The survey was completed by individuals in a variety of positions within the construction industry, including owners, vice presidents, BIM managers, BIM engineers, estimators, and architects.
Figure 1 compares the annual revenue of companies participating in the study and shows the percentage of survey participants that fall under each range of annual revenue.

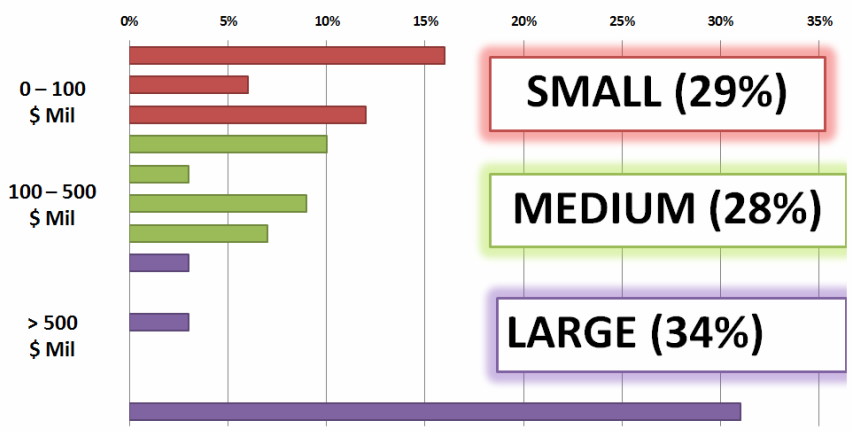

Figure 1: Annual Revenue of Companies Participating in the Study

Thirty-four percent of the participants worked for construction companies that annually generated greater than one billion dollars in revenue. Twenty nine percent of the survey participants worked for companies that annually generated less than one hundred million dollars in revenue. Twenty eight percent of survey participants work for a company with annual revenue in excess of one-hundred million dollars but less than five hundred million dollars. In this study, survey participants were categorized by company size, as shown in Figure 1. The participants in this survey have worked on projects in all aspects of the construction industry: education, government, healthcare, industrial, office, parking, religious, retail, and sports and leisure.

\section{RESULTS}

An important step in this study is to establish how general contractors are using Building Information Modeling in the construction industry. As shown in Figure 2, the survey participants were asked how they use BIM tools in their company. The graph shows what percentage of survey participants selected each item, as participants selected all applicable answers. 


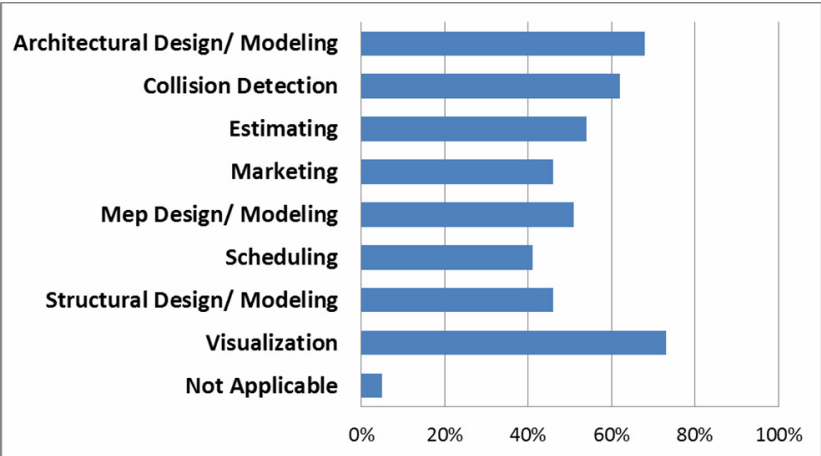

Figure 2: Uses for BIM Software in US Construction

The most common use of BIM is for visualization. Seventy-three percent of survey participants selected that they implement BIM for visualization, which is one of the most basic uses of Building Information Modeling. The design and modeling of the architectural model is the second most common use of BIM. The third most common use of Building Information Modeling, collision detection, is effective in catching collision early in the design process. The collision detection ability of BIM can save time and money by detecting geometric clashes in the design phase, before construction begins. Fifty-two percent of survey participants selected that they use BIM for cost estimating, while six percent of survey participants do not use BIM for any purpose.

The results can be further analyzed by company size. Collision detection is the most common use of BIM among large companies. Eighty-five percent of large companies selected that they use BIM for collision detection. Over seventy-five percent of large companies selected that they use BIM for all of the listed activities except for MEP and Structural modeling. Medium size companies primarily use BIM for visualization, architectural modeling, collision detection, and estimating. Over ninety-percent of participants from medium size companies selected that they use BIM for visualization. Fifty-five percent of medium size companies selected that they use BIM the least for marketing, scheduling, and structural modeling. Small firms are rarely using BIM. Visualization is the most common use of BIM among small firms, as fifty-five percent of survey participants from small firms selected that they use BIM for visualization. At thirty-six percent, the next most common use of BIM among small firms was architectural design and collision detection.

The charts below compare the use of architectural, MEP, and structural models in the BIM environment. Figure 3 demonstrates a comparison of how often survey participants created and used their own model for quantity take-offs. The architectural model is most commonly used by general contractors for estimating, but the structural model is most commonly used in the industry. The values shown are percentages.

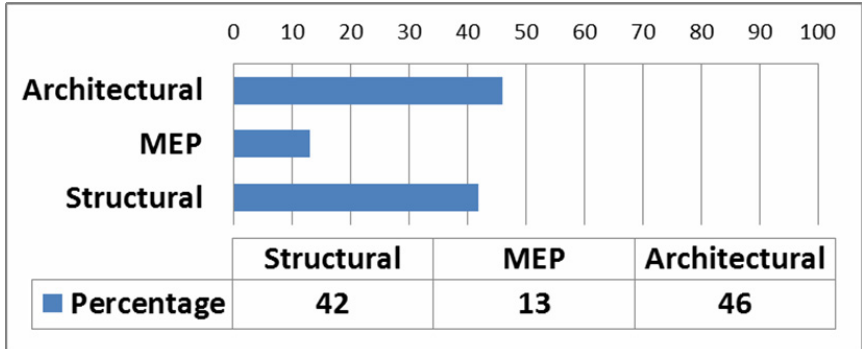

Figure 3: BIM Models created by US Contractors

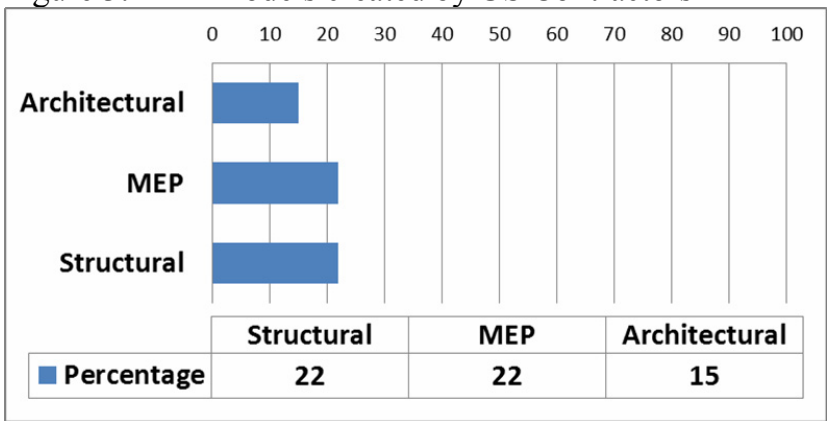

Figure 4: How Often Models Received from the Architect or Engineer Contain All Necessary Information to Perform a Quantity Take-off

General contractors often receive BIM models from architects, engineers, and subcontractors rather than designing their own models. Figure 4 compares how often the BIM models received from the architect or engineer contain all necessary information to perform a quantity take-off so that it can be used for estimating purposes. The values shown are percentages. Clearly the numbers indicate that contractors are unable to use the models for estimating purposes, as provided by their design counterparts. 
The construction industry is not going to move toward performing cost estimates with Building Information Modeling unless BIM is more beneficial than current estimating methods. Survey participants were asked if BIM has decreased the amount of time needed for cost estimating. Only forty-eight percent of the participants said BIM has decreased the amount of time needed for cost estimating. Survey participants were then asked if BIM has improved the quality of estimating. Sixty-nine percent selected that BIM has improved the quality of estimating. However, less than thirty percent of larger general contractors selected that BIM has improved the quality of estimating. One survey participant stated that BIM is not improving the quality of estimates, but expects this to change in the near future. All medium sized general contractors selected that BIM has improved the quality of estimating.

\section{CONCLUSIONS}

The future goal for Building Information Modeling is for general contractors to be able to perform a quantity takeoff in a few minutes and transfer it to estimating software, automating the cost estimating process. Currently, BIM is not increasing quality of estimates or decreasing the time required for cost estimating for large contractors. This could be the result of large general contractors having separate divisions for estimating and BIM or the result of large contractors primarily dealing with negotiated contracts. Most negotiated contracts are signed before design is complete and estimated conceptually; this hinders the feasibility of estimating with a detailed BIM model.

There needs to be an upgrade in BIM software before general contractors can efficiently estimate by applying durations and costs directly to the BIM model. Architects and design engineers need to creat BIM models with sufficient information so that cost estimates may be created without the contractor having to redraw entire models from scratch.

\section{REFERENCES}

[1] Aouad, G., Wu, S. and Lee, A. (2006), nDimensional Modeling Technology: Past, Present and Future, Journal of
Computing in Civil Engineering, American Society of Civil Engineers, 151-153, May/June.

[2] Bedrick, J., \& Rinella, T. (2006). Report on Integrated Practice. American Institute of Architects. Retrieved from http://www.aia.org/aiaucmp/groups/aia/documents/pdf/aias 076760.pdf.

[3] Buckley, B. (2008). More BIM Benefits: Building Information Modeling Offers a More Efficient way to Estimate Jobs. Building Information. 58.

[4] Facility Information Council. (2007), "National BIM Standard", Technical Report, National Institute of Building Sciences.

[5] Fischer, M. and Kunz, J. (2004), The Scope and Role of Information Technology in Construction, Proceedings Japan Society of Civil Engineers, Dotoku Gakkai, Japan, ISSU 763 1-32.

[6] Gier, D. M. (2007). What Impact Does Using Building Information Modeling Have on Teaching Estimating to Construction Management Students? Retrieved from http://ascpro0.ascweb.org/archives/cd/2008/paper/CEUE17 9002008.pdf.

[7] Hardin, B. (2009). BIM and Construction Management: Proven Tools, Methods, and Workflows. Indianapolis: Wiley.

[8] Howard R., \& Bjork, B. C., (2007). Building Information Modeling - Experts' views on standardization and industry deployment. Advanced Engineering Informatics. 271-280.

[9] Ibrahim, M., Krawczyk, R., \& Schipporeit, F. (2004). Two Approaches to BIM: A Comparative Study. Retrieved from www.itt.edu

[10] Kim, G. H., Yoon, J. E., An, S. H., Cho, H. H., \& Kang, K. I. (2004). Neural network model incorporating a genetic algorithm in Estimating Construction Costs. Building and Environment, 39, 1235-1242.

[11] Mihindu, S., Arayici, Y. (2008), Digital Construction through BIM Systems will drive the Re-engineering of Construction Business Practices, Proceedings: Visualisation, 2008 International Conference, IEEE, Place, v-vi, July.

[12] Sacks, R., Eastman, C. M., Lee, G., \& Orndorff, D. (2005). A Target Benchmark of the Impact of ThreeDimensional Parametric Modeling in Precast Construction. 
PCI Journal, 50, 126-139.

[13] Tiwari, S., Odelson, J., Watt, A., \& Khanzode, A. (2009). Model Based Estimating to Inform Target Value Design. AECbytes. Retrieved from http://www.aecbytes.com/buildingthefuture/2009/ModelBa sedEstimating.html. 